\title{
Evaluando el liderazgo educativo centrado en los aprendizajes del alumnado
}

\author{
MARCOS SARASOLA BONETTI \\ CAROLINA DA COSTA* \\ Universidad de Deusto - España
}

Recibido el 01-10-2015; primera evaluación el 20-11-2015; segunda evaluación el 10-11-2015; aceptado el 05-05-2016

\section{Resumen}

La investigación indica que el liderazgo educativo afecta los resultados de la gestión de los centros educativos en general, y en particular, incide en los aprendizajes del alumnado. Este artículo presenta y analiza el VAL-ED (Vanderbilt Assessment of Leadership in Education) como un instrumento de evaluación de la gestión, a partir de la investigación sobre liderazgo centrado en los aprendizajes.

Palabras clave: VAL-ED, liderazgo centrado en los aprendizajes, evaluación.

\section{Evaluating learning centered school leadership}

\section{Abstract}

The research shows that school leadership influences school management results in general, and it particularly impacts student learning. This article presents and analyzes the VAL-ED (Vanderbilt Assessment of Leadership in Education) as a school leadership assessment tool that is based on the research on learning centered leadership.

Keywords: VAL-ED, learning centered leadership, assessment.

\footnotetext{
Doctor en Educación (Universidad de Deusto, Bilbao), magíster en Educación y especialista en Gestión de Centros Educativos (Universidad Católica del Uruguay), profesor de Ciencias Físicas. Formado en el Programa Seekers After Truth de terapias integrativas. Meditador Vipassana tal y como la enseńa S.N.Goenka en la tradición de Sayagyi U Ba Khin. Asesor para la adaptación de la Metodología CREA a la educación. Contacto: marsaras@ucu.edu.uy

** Consultor para la capacitación de los Directores de Unidades de Internación de personas privadas de libertad del Instituto Nacional de Rehabilitación para la Organización Internacional del Trabajo / Cinterfor. Maestra de Educación Común en Institutos Normales «María Stagnero de Munar» y «Joaquín R. Sánchez». Dirección de Inicial y Primaria. Colegio Idejo, Montevideo, desde 2014. Contacto: carolinadac@gmail.com
} 


\section{Avaliando a liderança educacional focado na aprendizagem dos alunos}

\section{Resumo}

A pesquisa indica que a liderança educacional afeta os resultados da gestáo das escolas em geral, e em particular afeta a aprendizagem do aluno. Este artigo apresenta e analisa o VAL-ED (Avaliação Vanderbilt de Liderança em Educação) como uma ferramenta de avaliação da gestão, a partir de pesquisas sobre liderança centrada na aprendizagem.

Palavras chave: VAL-ED, liderança centrado na aprendizagem, avaliação.

\section{INTRODUCCIÓN}

En los últimos años la OCDE ha puesto especial atención a la formación de líderes educativos (Pont, Nusche, Moorman \& David, 2008a, 2008b) y el informe TALIS 2013 (OECD, 2014) le dedica un capítulo completo con el título La importancia del liderazgo escolar. Desde el reconocimiento de la complejidad, como así también de la incidencia que tiene el liderazgo en los centros educativos, el informe establece algunas características de los líderes educativos: (a) desarrollan una visión y demuestran competencia para compartirla con los docentes, inspirándolos y motivando su desarrollo; (b) permanecen especialmente atentos a los nuevos conocimientos sobre la didáctica, la forma en que aprenden los estudiantes y las prácticas adecuadas para evaluar los aprendizajes; (c) gestionan hábilmente a las personas, en general retroalimentándolas y promoviendo su crecimiento profesional; (d) atienden al tratamiento y los vínculos con las familias y el entorno más próximo, manejando redes profesionales; y por último, (e) administran los recursos económicos. Es así que sugieren a los países que apuesten a la formación y el desarrollo de estos líderes en cuatro líneas de acción:

- Desarrollar programas formales de formación inicial para los líderes educativos antes de ocupar los cargos correspondientes.

- Proveer oportunidades y allanar los caminos para el desarrollo profesional continuo de los directivos.

- Fomentar el liderazgo distribuido de los directivos.

- Asegurar que los directivos son formados y tienen las oportunidades de desarrollar un liderazgo centrado en los procesos de enseñanza y aprendizaje. 
La gestión del centro educativo depende, en gran medida, del rol del director, de su capacidad de liderazgo, de aunar los esfuerzos de los diferentes protagonistas, docentes, estudiantes y comunidad educativa, en aras de lograr las metas comunes, previamente consensuadas y definidas (Moos \& Huber, 2007).

El conjunto de los factores de organización y funcionamiento, mantiene estrecha relación con factores propios de los procesos que se desarrollan en aula, que son los que dan la razón de ser del centro. Numerosos estudios internacionales y regionales, ratifican y verifican la importancia de esta dinámica de relación entre el sentimiento de satisfacción y la gestión del centro (Creemers \& Scheerens, 1997; Murillo \& Castilla, 2011; Sammons, Hillman \& Mortimore, 1995; Scheerens, 2004; Teddlie \& Reynolds, 2000). No menos importante es el papel de los líderes educativos en un horizonte no muy lejano de mayor autonomía, donde los directivos tomen decisiones curriculares, decisiones en cuanto a los objetivos de aprendizaje y cómo supervisar los procesos para alcanzarlos. En el informe Preparing Teachers and Developing School Leaders for the 21st Century. Lessons from around the World (Schleicher, 2012) se hace un llamado explícito a la formación de profesionales en la gestión con un fuerte liderazgo, como conclusión de la investigación sobre de qué manera es menester trabajar en este siglo XXI.

Los directores a menudo destinan poco tiempo al liderazgo pedagógico ya que las actividades administrativas y los emergentes tienden a ocupar gran parte del tiempo. Así los equipos directivos ven cómo el tiempo se les va de entre las manos realizando tareas administrativas, atendiendo problemas edilicios, cumpliendo con exigencias externas, alejados de los temas pedagógicos le dan la espalda al aprendizaje.

Una gestión que busca acercarse a un enfoque de educación centrada en los aprendizajes necesita apoyarse en instrumentos, herramientas y conceptos que le ayuden a alinear sus prácticas en un plan de trabajo coherente con ese enfoque. La investigación en liderazgo educativo (Murphy, Elliott, Goldring $\&$ Porter, 2006) han logrado identificar prácticas de gestión que hacen que toda la institución se acerque a los objetivos de una educación centrada en los aprendizajes. Este artículo presenta y analiza el VAL-ED (Vanderbilt Assessment of Leadership in Education) como un instrumento de evaluación de la gestión concebido a partir de la investigación sobre liderazgo centrado en los aprendizajes. Este instrumento constituye un apoyo para los equipos de gestión que buscan liderar cambios en la manera en que se toman las decisiones colocando a los aprendizajes de los alumnos en el centro. 


\section{El liderazgo educativo: distribuido, para la JUSTICIA SOCIAL Y CENTRADO EN LOS APRENDIZAJES}

Si se analizan diferentes documentos que abordan el liderazgo educativo y que lo definen, es posible encontrar dos conceptos comunes a ellos: «influencia» y «direccionalidad». Algunos ejemplos pueden verse a continuación:

Marcar una meta común e influir en otros para compartirla (Leithwood \& Seashore-Louis, 2011).

Movilizar e influir en otros para desarrollar comprensiones compartidas acerca de las metas a alcanzar por la escuela (Firestone \& Riehl, 2005).

Influencia no basada en la fuerza, la coerción, la manipulación. Proviene del ejercicio razonable de la autoridad formal, de las propias cualidades del líder, de su grado de conocimiento y experiencia como para poder ofrecer orientaciones a los demás (Robinson, 2011).

Efecto mediado por el trabajo que realizan los educadores. La creación de un ambiente y de unas condiciones de trabajo que favorezcan a su vez un buen trabajo en las aulas es algo en lo que pueden incidir los equipos directivos (Bolívar, López-Yáñez \& Murillo Torrecilla, 2014).

Es decir, conceptualmente en este artículo, el liderazgo educativo se entiende como la competencia a través de la que las personas influyen planificada e intencionadamente en otros, con la finalidad de mejorar los aprendizajes del alumnado. De esta manera, el rol del director del centro educativo es fundamental a la hora de hacer una diferencia en el rendimiento académico de los estudiantes. El liderazgo escolar influye en los aprendizajes del alumnado a través de cuatro canales identificados por Leithwood, Anderscon, Mascall y Strauss (2010): el racional, el emocional, el organizacional y el familiar. En cada uno de estos canales existen variables que influyen en los aprendizajes de los alumnos de diferentes maneras. Las mejoras en la gestión educativa deberán entonces tener en cuenta estos canales y variables para lograr mejoras en los aprendizajes. Las prácticas de gestión que forman la base para un centro educativo donde los alumnos logren aprendizajes significativos, se desprenden de las características del liderazgo efectivo (Leithwood, Seashore Louis, Anderson \& Wahlstrom, 2004).

El liderazgo en los centros educativos es considerado fundamental en los procesos de cambio: es el segundo factor después del trabajo en el aula, que incide en los aprendizajes de los alumnos (Leithwood et al., 2004). Más recientemente Bolívar Batía (2012) hace una excelente revisión de la literatura internacional y de experiencias que muestran la relevancia del liderazgo en tanto factor de impacto en los aprendizajes. Esta línea ha sido retomada en el marco del Plan Nacional I+D+i de España, centrándose en tres dimensiones 
de la investigación actual sobre liderazgo: el liderazgo pedagógico, el liderazgo distribuido y el liderazgo para la justicia social (Bolívar et al., 2014), a las que se hará referencia somera a continuación.

\section{El liderazgo distribuido}

Existe un cierto consenso en la literatura del liderazgo acerca de la necesidad de un liderazgo distribuido (Gronn, 2002; Spillane, Halverson \& Diamond, 2001, 2004). El interés en este tipo de liderazgo se sustenta en la evaluación de las prácticas de las personas que ocupan cargos directivos y académicos en el centro. Esta perspectiva es consistente con los argumentos expuestos por Locke (2003) en sus modelo de liderazgo distribuido que sostienen Leithwood, Mascall, Strauss, Sacks, Memon y Yashkina (2007).

No obstante y aunque resulta patente la necesidad de distribuir el liderazgo, la distribución del liderazgo encuentra resistencias aun cuando el director no puede abordar por sí mismo todas las decisiones que tienen que tomarse en su centro, ni supervisar todos los procesos, o estar presente cuando los docentes tienen que evaluar o resolver conflictos en el aula.

El conocimiento disponible actualmente acerca de los factores que influyen en el desarrollo del liderazgo distribuido en los centros educativos, es el producto de la investigación en las dos últimas décadas. Los hallazgos obtenidos indican que el grado en que los docentes asumen funciones de liderazgo organizativo depende de las características de la estructura y cultura del centro, la naturaleza de las relaciones dirección-docentes, y el apoyo a las formas distribuidas de liderazgo por parte de los directores (Day \& Harris, 2002; Harris, 2005; Harris \& Lambert, 2003; Leithwood, Jantzi, Steinbach, Bennett, Crawford \& Cartwright, 2003; Leithwood et al., 2007; MacBeath, 2005; Smylie, Conley \& Marks, 2002).

Uno de los aspectos más destacables extraído de un estudio realizado por Leithwood et al. (2007) sobre la distribución del liderazgo en una muestra de escuelas de un distrito urbano del sur de Ontario, es el hallazgo relativo al papel fundamental desempeñado por los líderes formales del centro educativo y del distrito en contribuir a desarrollar formas productivas de liderazgo distribuido. Los directores dieron prioridad a modelos coordinados planificados de liderazgo distribuido. Asimismo, los resultados del estudio refuerzan el papel de los directores de los centros, en cuanto que se observó que las estructuras, normas culturales y oportunidades para que el personal docente desarrollase sus capacidades de liderazgo, dependían profundamente del trabajo intencionado hecho por los directores (Leithwood et al., 2007). 
Smylie, Conley y Marks (2002) realizaron una revisión de la investigación sobre el liderazgo distribuido y el liderazgo del docente. Como conclusión de su trabajo, los autores subrayan dos ideas muy importantes que dan luz a esta cuestión de los nuevos enfoques de liderazgo. Una de las ideas es la importancia de la figura del director y del liderazgo formal del director para el funcionamiento y los resultados de los nuevos enfoques de liderazgo. El liderazgo del director, explican los autores, sirve de ejemplo, incentivo, guía, apoyo y como medio para la responsabilidad ante los resultados.

Una segunda idea que subrayan los investigadores como conclusión a la revisión, es la necesidad de que la organización más amplia y los contextos institucionales en los que se desarrollan y operan los nuevos enfoques de liderazgo sean favorables para que las iniciativas de liderazgo puedan llegar a ser eficaces: descubrir y promover las condiciones que permiten que el proceso de liderazgo florezca.

\section{El liderazgo para la justicia social}

El concepto de educación social sigue presentando algunas dificultades tanto en su aproximación conceptual, como en la forma que puede desarrollarse en las sociedades (Bogotch, 2008). El impacto que puede conseguir un centro educativo que trabaja con una población vulnerable, es mayor que el impacto logrado por los centros educativos con una población que no lo es, como ya ha sido medido. Concretamente los estudios de Louis (2010) y de Robinson, Hohepa \& Lloyd (2007) coinciden en destacar la importancia del liderazgo pedagógico en los centros educativos para incrementar la calidad de los aprendizajes. Es más, hoy se sabe que sus efectos son aún mayores en contextos de población vulnerable como afirma Bolívar (2009) a partir de los resultados del metaestudio de Waters, Marzano y McNulty (2003).

Los centros educativos pueden facilitar al alumnado más vulnerable, el desarrollo de una estructura social y emocional que no suelen haber recibido en sus hogares. Pueden, sin dudas, aunque no es lo más generalizado. La buena noticia es que sucede y allí hay conocimiento acumulado: existen centros que logran ayudar a superar esta situación desventajosa (Aristimuño \& Lasida, 2003; Barr \& Parrett, 2007; Harris, Clarke, Gunraj, James \& James, 2006). Lo que no es muy alentador es la lentitud con la que se logran las mejoras y el trabajo duro y desgastante que supone alcanzarlas. Intentar la aplicación del conocimiento que hoy existe sobre el cambio y la innovación en centros educativos, en aquellos que trabajan con una población vulnerable, es completamente inadecuado. La experiencia así lo demuestra (Harris, 2009). 
Desde la perspectiva de la justicia social, se abre un camino para desarrollar prácticas de liderazgo con una especial conciencia. En una aproximación al concepto actual de justicia social (Murillo Torrecilla \& Hernández-Castilla, 2011), es posible establecer cuatro dimensiones, no excluyentes, sino que vinculadas y complementarias y que podrían sinterizarse en: responsabilidad, redistribución, reconocimiento y representación.

- Responsabilidad. En un artículo con el sugestivo nombre de La guerra contra la pobreza debe ganarse: los líderes transformativos pueden marcar la diferencia (Shields, 2014), se encuentran evidencias del impacto que pueden tener los líderes. Es un llamado de atención a la responsabilidad de los educadores y de lo que pueden hacer frente a la inequidad.

- Redistribución. No solo entendida como la redistribución de recursos y bienes primarios, sino también como dar más a quien por sus condiciones o su situación de partida más lo necesita.

- Reconocimiento y valoración de las diferencias cultuales, sociales y personales.

- Representación, como participación real de todos los grupos, especialmente de aquellos tradicionalmente excluidos.

No obstante, vale la pena tomar los recaudos necesarios frente a posibles simplismos o perspectivas ingenuas sobre la justicia social. Las dificultades ya mencionadas al principio de este apartado, se refuerzan en el análisis que hace da Silveira (2009) sobre la igualdad de oportunidades y el gobierno de la educación.

Ahora bien, volviendo al liderazgo y su concreción en la práctica, es necesario traducir a la práctica estas características del liderazgo para la justicia social. Por el momento, en un nivel de hipótesis, se han formulado una serie de prácticas (Bolívar et al., 2014) que están profundamente relacionadas a las prácticas identificadas por diversos autores como, por ejemplo, Leithwood (2010), a las que se enriquece en algunos casos enfatizando la justicia social. En este sentido, vale destacar las siguientes prácticas:

- Identificar y articular una visión del centro con foco en la justicia social.

- Potenciar una cultura de centro en y para la justicia social.

- Los miembros de la comunidad escolar y su desarrollo personal, social y profesional, son la máxima prioridad.

- Centrarse en la mejora de los procesos de enseñanza y aprendizaje.

- Potenciar la creación de comunidades profesionales de aprendizaje. 
- Promover la colaboración entre la escuela y la familia, potenciando el desarrollo de culturas educativas en las familias.

- Expandir el capital social de los estudiantes valorizado por los centros educativos.

\section{El liderazgo centrado en los aprendizajes}

El liderazgo centrado en los aprendizajes busca que el aprendizaje de los alumnos sea una prioridad en todos los niveles y todos los ambientes del centro educativo. El aprendizaje, la enseñanza, el currículo y las evaluaciones son el centro de la actividad del director, quien se asegura que las dimensiones organizacionales y administrativas estén al servicio del aprendizaje (Goldring, Porter, Murphy, Elliott \& Cravens, 2009). Una mejora en la calidad de los centros educativos estará enmarcada en las dimensiones de un liderazgo centrado en los aprendizajes (Murphy, Elliott, Goldring \& Porter, 2006): visión de aprendizaje, programa de enseñanza, programa curricular, plan de evaluaciones, comunidades de aprendizaje, uso de recursos, cultura organizacional y promoción social. La evaluación del liderazgo forma parte de un proceso de reflexión hacia un modelo de liderazgo que promueve el aprendizaje efectivo en la escuela.

Desde los seguidores de la corriente del liderazgo centrado en los aprendizajes, se ha buscado la forma de definir cuáles serían las prácticas propias de este liderazgo, con la finalidad de detectarlas en el ejercicio profesional. Esta idea está vinculada con evaluar, es decir comparar, el desempeño profesional de líderes de un centro educativo, mediante un instrumento que permita detectar brechas con el deber ser. Una de estas herramientas es el Vanderbilt Assessment of Leadership in Education, que se desarrollarán en las página siguientes.

\section{VAL-ED}

El Vanderbilt Assessment of Leadership in Education (en adelante VAL-ED) es un instrumento de $360^{\circ}$ para la evaluación del liderazgo centrado en los aprendizajes que fue desarrollado en la Universidad de Vanderbilt, en el marco de un proyecto con fondos de Wallace Foundation y Department of Education/ Institute for Education Science, a cargo de los investigadores Andrew Porter (University of Pennsylvania), Joseph Murphy (Vanderbilt University), Ellen Goldring (Vanderbilt University) y Stephen Elliot (Vanderbilt University). VAL-ED mide el desempeño del líder del centro educativo y puede ser utilizado como herramienta de evaluación integral además de tener como fin 
«obtener perfiles diagnósticos con propósitos formativos» (Murphy, Goldring, Cravens, Elliott \& Porter, 2007, p. 24). Este instrumento permite evaluar las prácticas de gestión desde un marco de educación centrada en los aprendizajes del alumnado como insumo para la mejora de la gestión. Los directores pueden entonces planear mejoras en la gestión tomando como insumo los resultados del VAL-ED. El instrumento guarda coherencia con los estándares del Interstate School Leaders Licensure Consortion (ISLLC).

VAL-ED recoge las respuestas del supervisor (inspector), el director y los docentes sobre 72 comportamientos de liderazgo que se enmarcan en seis componentes básicos y seis procesos clave para un liderazgo centrado en los aprendizajes de los alumnos (Goldring et al., 2009). Para cada uno de los 72 ítems, cada persona que responde va a valorar el grado de efectividad del director que puede encontrarse entre los conceptos de «no hay efectividad» $y$ «efectividad excepcional». La valoración otorgada a cada práctica va a estar en concordancia con la evidencia sobre esa práctica. Antes de responder sobre el grado de efectividad de una práctica, quien responde al cuestionario buscará evidencia que respalde su juicio. Cada respuesta estará entonces basada en fuentes de evidencia como observaciones personales, información recogida de otras personas y documentos.

Este cuestionario cuenta con evidencia sobre su validez y fiabilidad (Goldring, Cravens, Murphy \& Porter, 2012). A los efectos de cuidar estos dos aspectos, es decir, que mida aquellas prácticas del liderazgo relacionadas con los aprendizajes (validez) y que la medida sea precisa (fiabilidad), la evidencia psicométrica es sustancial; han desarrollado diversos procesos que han incluido trabajo en laboratorio, evaluación de piloto y en campo. En cada etapa de diseńo y desarrollo de los procesos, las características del instrumento fueron analizadas empíricamente así como a través de la revisión de expertos. Todo el proceso fue guiado por los American Educational Research Association's 1999 Standards for Educational and Psychological Testing. De esta manera se pretende que: (a) funcione bien en una variedad de entornos y circunstancias, (b) sea un constructo válido, (c) sea fiable, (d) sea imparcial; (e) proporcione información precisa y presente, (f) los diagnósticos ayuden a la formación, (g) permita medir el progreso a lo largo del tiempo en el desarrollo del liderazgo y (h) prediga los resultados más sustantivos (Murphy et al., 2007).

Desde el punto de vista conceptual y estructural, se divide en componentes básicos (qué deben hacer los directores o equipos de liderazgo para mejor el aprendizaje académico y social de los alumnos) y procesos clave (cómo llegar a estos componentes básicos). De ahí que sea vital analizar la intersección entre unos y otros, como se verá a continuación. 


\section{Componentes básicos}

Según Porter, Murphy, Goldring, Elliott, Polikoff y May (2008) hay seis características de los centros educativos efectivos que apoyan realmente al aprendizaje del alumnado y promueven buenas prácticas de los docentes. Estas son:

- Altos niveles de logro en los aprendizajes del alumnado

El liderazgo efectivo confía en plantearse objetivos de aprendizaje, claros y exigentes, en cuanto a los logros académicos esperables para todos los estudiantes.

- Currículo exigente

El currículo ofrece oportunidades para que todos los estudiantes estén expuestos a contenidos académicos ambiciosos en todas las asignaturas. Además el liderazgo efectivo garantiza que todos y cada uno de los estudiantes puedan aprovechar el currículo al máximo.

- Educación de calidad

Un excelente currículo necesita además una excelente práctica docente. La gestión efectiva garantiza el uso de estrategias pedagógicas que tienen como resultado mejores aprendizajes académicos y sociales, demostrando un conocimiento sobre la manera en que aprenden las personas.

- Cultura de aprendizaje y práctica profesional

Una cultura de comunidad profesional que está centrada los aprendizajes, donde se valora la colaboración, el diálogo reflexivo y una visión compartida. Es decir, un ambiente de trabajo que prioriza el aprendizaje tanto de los docentes como de los alumnos.

- Vínculos con el entorno

La gestión busca que el centro tenga una relación sólida y positiva con las familias y con la comunidad externa, estableciendo y manteniendo canales de comunicación y colaboración con ellos.

- Responsabilidad por el desempeño

El liderazgo efectivo explicita las responsabilidades individuales y colectivas con respecto a los logros académicos de los estudiantes, en un clima de apoyo y colaboración.

\section{Procesos clave}

Los directores se relacionan con los componentes básicos antes mencionados, a través de seis procesos claves. Estos son: 


\section{- Planificación}

Planificación de cada uno de los componentes básicos como una manera de mantener las prioridades y alcanzar los objetivos en el aprendizaje de los alumnos. La gestión centrada en los aprendizajes entiende a la planificación como un mecanismo facilitador hacia el logro académico de los alumnos y participa activamente de ella.

\section{- Implementación}

Los directores llevan a la práctica las acciones tomando una actitud proactiva, modelando, implementando las actividades planificadas.

\section{- Apoyo}

El apoyo de los directores implica dedicación y tiempo destinado a crear las condiciones para el efectivo desarrollo de las actividades. Fundamentalmente se trata del apoyo a los docentes facilitándoles el acceso a recursos materiales y a oportunidades para la mejora de su práctica.

\section{- Promoción}

El director defiende los intereses y necesidades de los estudiantes asegurando la inclusión de manera que todos tengan oportunidades para aprender. Busca que las diferencias individuales entre estudiantes no se transformen en barreras para el aprendizaje.

\section{- Comunicación}

Una comunicación eficaz a la interna de la comunidad educativa y con la comunidad externa que garantiza una articulación entre los objetivos escolares y las prácticas educativas.

\section{- Supervisión}

Rol activo del director garantizando que las acciones planificadas se lleven a cabo. Para ello, recoge datos de la práctica docente y toma decisiones basado en evidencias fiables.

Las prácticas de gestión efectivas de los líderes del centro educativo se encuentran ubicadas en el cruce de estos seis componentes básicos y seis procesos clave. Las prácticas puntualizadas en los 72 ítems del VAL-ED tienen como resultado una mejora en el desempeño del centro educativo, de esta manera se logran mejoras en los resultados académicos del alumnado.

Como se muestra en la Tabla 1, cada uno de los componentes básicos (columna de la tabla) representa las dimensiones del liderazgo de la dirección y cada uno supone procesos (fila superior) de la tabla. 


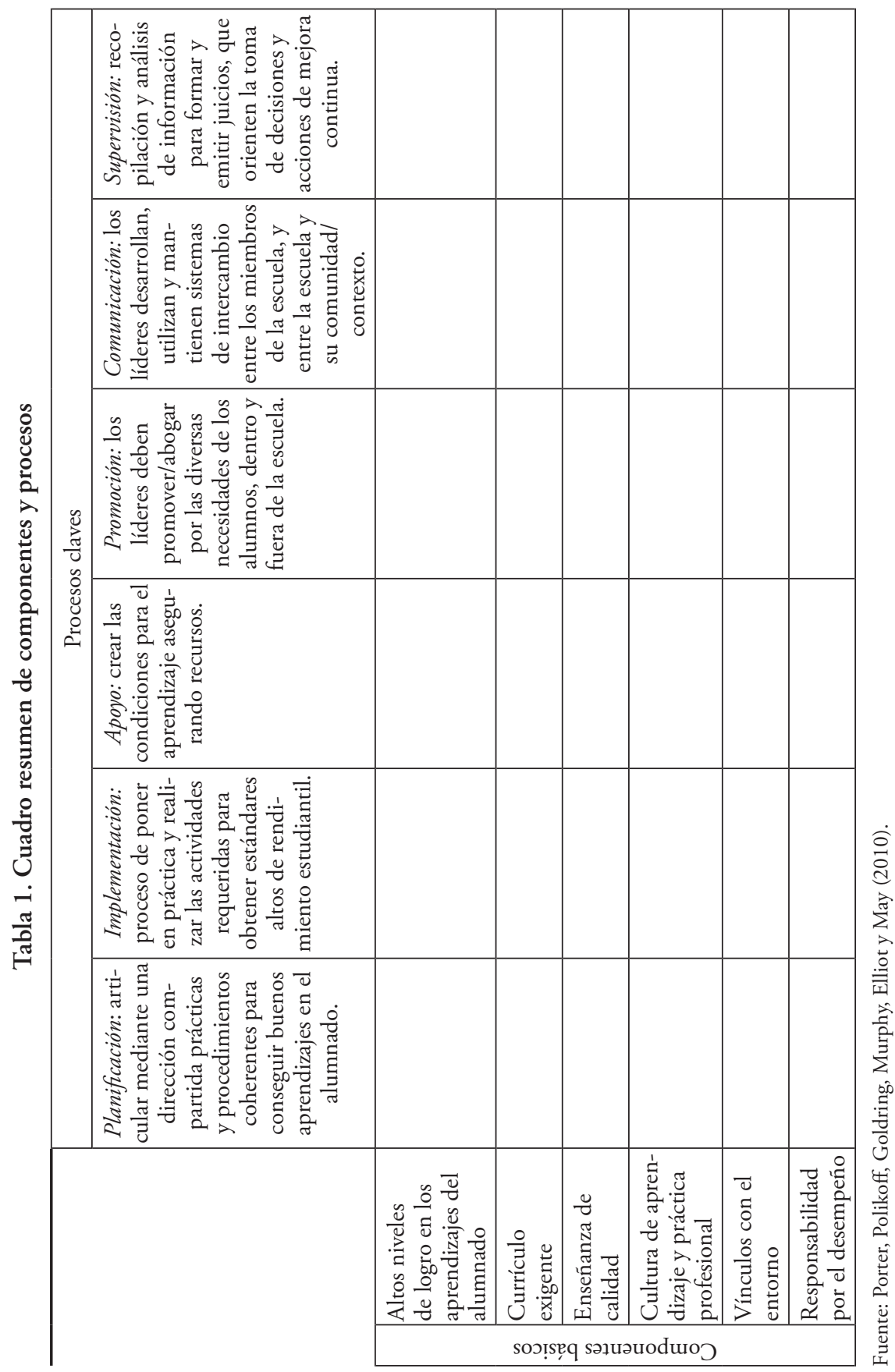




\section{Aplicar el VAL-ED de manera efectiva}

La reflexión sobre la práctica es, según Fullan (2011), la clave para una gestión que aprende y que sostiene los cambios. Desde esta visión del cambio como algo que sucede en y para la práctica, el líder en educación aprende reflexionando, aprende haciendo. Analizar la propia práctica puede transformarse en una tarea que sobrepasa a la dirección por las muchas y complejas dimensiones que se abarcan en la tarea de gestión. Es necesario guiar esa reflexión para hacerla posible utilizando herramientas como el análisis y la evaluación del proyecto institucional, contrastar la práctica con la normativa vigente, orientando las instancias formativas que complementan la tarea de gestión, o el apoyo en un instrumento de evaluación de la gestión.

La evaluación del liderazgo utilizando el instrumento VAL-ED inicia un cambio positivo en el centro que lleva a mejores niveles de desempeńo del alumnado. VAL-ED es una herramienta para apuntalar la gestión a través de un análisis y una reflexión sobre la práctica cotidiana, que permite identificar áreas de la gestión que necesitan un plan de trabajo con objetivos de mejora. De acuerdo a los resultados obtenidos en la evaluación se diseña un plan de mejora de la gestión desde un enfoque de educación centrada en los aprendizajes. De esta manera se logra coherencia entre una visión de educación centrada en los aprendizajes y las prácticas de gestión, concretas y cotidianas.

El VAL-ED cumple la función de la evaluación de la gestión desde el enfoque de educación centrada en los aprendizajes, con lo cual el foco de la reflexión se hace concretamente sobre las prácticas relevantes para un liderazgo efectivo. La incorporación del VAL-ED al quehacer de la gestión es un desafío que requiere tiempo y atención. Es necesario tomarse el tiempo para conocer los objetivos y el procedimiento de la evaluación, de manera que tanto la dirección del centro educativo como los docentes y los supervisores deberán prever instancias de familiarización con el VAL-ED, con su vocabulario y el alcance de sus conceptos.

El centro educativo que cuenta con un plan de evaluación de la gestión puede incorporar al VAL-ED como un instrumento para la mejora de la práctica y no tiene un carácter normativo o punitivo, por lo cual se utiliza con honestidad profesional y apertura personal. De esta manera la reflexión y el cambio sobre una práctica que parece inabarcable al principio se vuelve posible, enriqueciendo la gestión desde el momento en que se comienza a utilizar hasta el análisis de los resultados.

El centro educativo incorpora el VAL-ED en la evaluación de su gestión previendo y facilitando las siguientes actividades: 
- Presentación del VAL-ED en el centro educativo. Incluyendo una instancia de preguntas y respuestas sobre los ítems con el fin de familiarizar al personal docente, al director y al supervisor del director, con el vocabulario y los conceptos del instrumento.

- Sesión para completar el cuestionario. El cuestionario VAL-ED fue diseńado para ser respondido por tres grupos de personas: por un lado los docentes que trabajan en el centro, por otro lado por el propio director que realiza una autoevaluación, y finalmente por el supervisor del director. La instancia de aplicación del instrumento será cerca de la mitad del año escolar cuando todos los docentes están familiarizados con la gestión del director. Está previsto que completar el cuestionario tome alrededor de una hora si las personas han tenido oportunidad de conocer el instrumento y aclarar sus dudas.

- Periodo de análisis de los resultados. Se procesan todas las respuestas obtenidas y se organiza la información de acuerdo a las escalas de efectividad en liderazgo centrado en los aprendizajes manejadas en el diseño del VAL-ED.

- Presentación de los resultados en el contexto del centro. Una vez finalizado el análisis, se organiza una instancia de devolución al centro educativo en la que se orienta al equipo de trabajo sobre el significado de los resultados obtenidos y las posibles acciones a tomar a partir de ellos.

- Plan de apoyo a la mejora. De acuerdo a los resultados del VAL-ED y de acuerdo al análisis contextual de esos resultados en el marco de las necesidades y posibilidades específicas del centro educativo, se organiza un plan de mejora de la gestión. El plan de mejora es una manera concreta de ordenar y priorizar las necesidades de la gestión apoyándose en las fortalezas ya existentes.

\section{Conclusión}

El VAL-ED prioriza la evaluación de la gestión desde un enfoque de liderazgo centrado en los aprendizajes a través de seis procesos claves y seis componentes básicos apuntando a la mejora. La traducción del VAL-ED al español y la adaptación del instrumento realizadas en la Universidad de Granada (Bolívar, Caballero, López \& Pérez García, 2012; Bolívar, López, Pérez García \& García, 2012), posibilita su aplicación en países de habla hispana. Ha de advertirse, sin embargo, que la adaptación intercultural es necesaria, si el instrumento se quiere aplicar a una población objetivo culturalmente diferente 
a la de la versión original. Esto, seguramente, podrá requerir la modificación o eliminación de elementos de la escala o cuestionario original. Por tanto, la traducción es solamente el primer paso en el proceso de adaptación.

La incorporación del VAL-ED como instrumento de evaluación del liderazgo centrado en los aprendizajes, implica una disposición del centro educativo para completar un proceso de evaluación y mejora que supone el desarrollo de otras acciones. La aplicación del cuestionario se formula como la primera de ellas, de tal suerte que permita detectar prácticas cuyo efecto en los aprendizajes se pueda evidenciar (Bolívar Botía, 2014).

\section{REFERENCIAS BIBLIOGRÁFICAS}

Aristimuño, A. \& Lasida, J. (2003). Políticas y estrategias para el mejoramiento de las oportunidades de los jóvenes. Estudio sobre la educación secundaria en Uruguay. París: IIPE-UNESCO.

Barr, R. D. \& Parrett, W. H. (2007). The kids left behind: Catching up the underachieving children of poverty. Bloomington, IN: Solution Tree Press.

Bogotch, I. (2008). Social justice as an educational construct. En F. B. I. Bogotch, J. Blount, J. Brooks, J. Brooks y F. English (Eds.), Radicalizing educational leadership: Dimensions of social justice (pp. 79-112). Rotterdam y Taipei: Sense Publishers.

Bolívar, A. (2009). Una dirección para el aprendizaje. REICE: Revista Electrónica Iberoamericana sobre Calidad, Eficacia y Cambio en Educación, 7(1), 1-4.

Bolívar, A., Caballero, K., López, M. C. \& Pérez García, P. (2012). Assessing educational leaders: validation and adaptation of the Spanish version of $V A L-E D$. Paper presented at the Conferencia Europea sobre Investigación Educativa «The need for Educational Research to Champion Freedom, Education and Development for All, Cadiz. http://www.eera.de/ecer2012/

Bolívar, A., López-Yáñez, J. \& Murillo Torrecilla, F. J. (2014). Liderazgo en las instituciones educativas: Una revisión de líneas de investigación. Revista de la Facultad de Ciencias de la Educación de la Universidad de Sevilla, (14), $15-40$.

Bolívar, A., López, M. C., Pérez García, P. \& García, M. (2012). Medir el liderazgo pedagógico: Validación y adaptación al contexto español del VAL-ED. Paper presented at the Congreso Asociación Mundial de la Educación, Reims. http://www.univ-reims.fr/amse201

Bolívar Botía, A. (2012). ¿Cómo un liderazgo pedagógico y distribuido mejora los logros académicos? Revisión de la investigación y propuesta. Magis. Revista Internacional de Investigación en Educación, 3(5). 
Bolívar Botía, A. (2014). Building School Capacity: Shared Leadership and Professional Learning Communities. A Research Proposal. IJELMInternational Journal of Educational Leadership and Management, 2(2), $147-175$.

Creemers, B. P. M. \& Scheerens, J. (1997). Developments in the educational effectiveness research programme. International Journal of Educational Research, 21, 125-140.

da Silveira, P. (2009). Padres, maestros, y politicos: el desafio de gobernar la educación. Buenos Aires: Taurus.

Day, C. \& Harris, A. (2002). Teacher leadership, reflective practice, and school improvement. Second International Handbook of Educational Leadership and Administration (pp. 957-977). Springer.

Firestone, W. A. \& Riehl, C. (2005). A new agenda for research in educational leadership. Nueva York: Teachers College Press.

Fullan, M. (2011). Change leader: Learning to do what matters most. Nueva York: John Wiley \& Sons.

Goldring, E., Cravens, X., Murphy, J. \& Porter, A. (2012). The convergent and divergent validity of the Vanderbilt Assessment of Leadership in Education $^{\mathrm{Tm}}(V A L-E D)$ : Instructional leadership and emotional intelligence. Paper presented at the Annual Conference of Association for Education Finance and Policy, Boston, MA.

Goldring, E., Porter, A., Murphy, J., Elliott, S. N. \& Cravens, X. (2009). Assessing learning-centered leadership: Connections to research, professional standards, and current practices. Leadership and Policy in Schools, 8(1), 1-36.

Gronn, P. (2002). Distributed leadership. Second International Handbook of Educational Leadership and Administration (pp. 653-696). Springer.

Harris, A. (2005). Crossing boundaries and breaking barriers. Londres: Specialist Schools Trust.

Harris, A. (2009). Improving Schools in Challenging Contexts Second International Handbook of Educational Change (pp. 693-706). Springer.

Harris, A., Clarke, P., Gunraj, J., James, B. \& James, S. (2006). Improving schools in exceptionally challenging circumstances: Tales from the frontline. Londres: Continuum.

Harris, A. \& Lambert, L. (2003). Building leadership capacity for school improvement. Nueva York: McGraw-Hill International.

Leithwood, K., Anderson, S. E., Mascall, B. \& Strauss, T. (2010). School leaders' influences on student learning: The four paths. En T. Bush, L. Bell y D. Middlewood (Eds.), The principles of educational leadership and management (pp. 13-30). Londres: Sage. 
Leithwood, K., Jantzi, D., Steinbach, R., Bennett, N., Crawford, M. \& Cartwright, M. (2003). Fostering teacher leadership. En N. Bennet, M. Crawford y M. Cartwright (Eds.), Effective educational leadership (pp. 186-200). Londres: Paul Chapman.

Leithwood, K., Mascall, B., Strauss, T., Sacks, R., Memon, N. \& Yashkina, A. (2007). Distributing leadership to make schools smarter: Taking the ego out of the system. Leadership and Policy in Schools, 6(1), 37-67.

Leithwood, K. \& Seashore-Louis, K. (2011). Linking leadership to student learning. Hoboken, NJ: John Wiley \& Sons.

Leithwood, K., Seashore Louis, K., Anderson, S. \& Wahlstrom, K. (2004). Review of research: How leadership influences student learning. Nueva York: The Wallace Foundation.

Locke, E. A. (2003). Leadership: Starting at the top. En C.L. Pearce y J.A. Conger, Shared leadership: Reframing the hows and whys of leadership (pp. 271-284). Thousand Oaks, CA: Sage.

Louis, K. S. (2010). Learning from leadership project: Investigating the links to improved student learning: Final report of research findings. St. Paul: MN: University of Minnesota, Center for Applied Research and Educational Improvement.

MacBeath, J. (2005). Leadership as distributed: a matter of practice. School leadership and management, 25(4), 349-366.

Moos, L. \& Huber, S. (2007). School leadership, school effectiveness and school improvement: democratic and integrative leadership International handbook of school effectiveness and improvement (pp. 579-596). Springer.

Murillo, J. F. \& Castilla, R. H. (2011). Factores escolares asociados al desarrollo socio-afectivo en iberoamérica Revista electrónica de investigación y evaluación educativa, 17(2), 1-23.

Murillo Torrecilla, F. J. \& Hernández-Castilla, R. (2011). Hacia un concepto de justicia social. REICE: Revista Electrónica Iberoamericana sobre Calidad, Eficacia y Cambio en Educación, 9(4), 7-23.

Murphy, J., Elliott, S. N., Goldring, E. \& Porter, A. C. (2006). Learning-Centered Leadership: A Conceptual Foundation. Nashville, TN: Vanderbilt University.

Murphy, J. F., Goldring, E. B., Cravens, X. C., Elliott, S. N. \& Porter, A. C. (2007). The Vanderbilt assessment of leadership in education: Measuring learningcentered leadership. Journal of East China Normal University, agosto.

Murphy, J. F., Goldring, E. B., Cravens, X. C., Elliott, S. \& Porter, A. C. (2011). The Vanderbilt Assessment of Leadership in Education: Measuring Learning-Centered Leadership. Journal of East China Normal University, 29(1), 1-10. 
OECD (2014). TALIS 2013 Results: An International Perspective on Teaching and Learning. OECD Publishing. http://dx.doi.org/10.1787/ 9789264196261-en

Pont, B., Nusche, D., Moorman, H. \& David, H. (2008a). Improving school leadership, Volume 1: Policy and practice. OECD Publishing.

Pont, B., Nusche, D., Moorman, H. \& David, H. (2008b). Improving School Leadership, Volume 2 Case Studies on System Leadership: Case Studies on System Leadership. OECD Publishing.

Porter, A. C., Murphy, J., Goldring, E., Elliott, S. N., Polikoff, M. S. \& May, H. (2008). Vanderbilt Assessment of Leadership in Education: Technical Manual, Version 1.0. Nashville, TN: Vanderbilt University.

Porter, A. C., Polikoff, M. S., Goldring, E., Murphy, J., Elliott, S. N. \& May, H. (2010). Developing a psychometrically sound assessment of school leadership: The VAL-ED as a case study. Educational Administration Quarterly, 46(2), 135-173.

Robinson, V. (2011). Student-centered leadership (Vol. 15). Hoboken, NJ: John Wiley \& Sons.

Robinson, V. M., Hohepa, M. \& Lloyd, C. (2007). School leadership and student outcomes: Identifying what works and why. Winmalee, Victoria, Australia: Australian Council for Educational Leaders.

Sammons, P., Hillman, J. \& Mortimore, P. (Eds.). (1995). Key Characteristics of Effective Schools: A Review of School Effectiveness Research. Londres: Office for Standards in Education.

Scheerens, J. (2004). Review of school and instructional effectiveness research. Contribution to chapter 3 of the 2004 EFA Global Monitoring Report. http://unesdoc.unesco.org/images/0014/001466/146695e.pdf.

Schleicher, A. (2012). Preparing Teachers and Developing School Leaders for the 21st Century Lessons from around the World: Lessons from around the World: OECD Publishing.

Shields, C. M. (2014). The War on Poverty Must Be Won: Educators Can Make a Difference. IJELM-International Journal of Educational Leadership and Management, 2(2), 124-146.

Smylie, M. A., Conley, S. \& Marks, H. M. (2002). Exploring new approaches to teacher leadership for school improvement. Yearbook of the National Society for the Study of Education, 101(1), 162-188.

Spillane, J. P., Halverson, R. \& Diamond, J. B. (2001). Investigating school leadership practice: A distributed perspective. Educational Researcher, 30(3), 23-28. 
Spillane, J. P., Halverson, R. \& Diamond, J. B. (2004). Towards a theory of leadership practice: A distributed perspective. Journal of curriculum studies, 36(1), 3-34.

Teddlie, C. \& Reynolds, D. (2000). The International Handbook of School Effectiveness Research. Londres y Nueva York: Falmer Press.

Waters, T., Marzano, R. J. \& McNulty, B. (2003). Balanced Leadership: What 30 years of research tells us about the effect of leadership on student achievement. Retrieved from www.mcrel.org/PDF/LeadershipOrganizationDevel opment/5031RR_BalancedLeadership.pdf 